\title{
VALORES CARACTERÍSTICOS DOS PARÂMETROS DE REFERÊNCIA DE EMISSÕES DOS VEÍCULOS BRASILEIROS
}

\author{
Fábio Cardinale Branco ${ }^{1}$ \\ Gabriel Murgel Branco² \\ Alfred Szwarc ${ }^{3}$ \\ Luiz Vicente Figueira de Mello Filho ${ }^{4}$ \\ José Mauro Napoleone ${ }^{5}$
}

\section{Resumo}

Este trabalho apresenta uma metodologia estatística para a identificação dos valores característicosefetivamente observadosnos veículos em uso, aprovados no Programa de Inspeção e Manutenção - I/M-SP e que podem ser utilizados comoreferência em outros Programas $\mathrm{I} / \mathrm{M}$, para verificação da conformidade de emissões dos veículos em uso com as especificações originaisdo fabricante.

Foram determinados três níveis de cada parâmetro de emissão, com base no banco de dados do Programa I/M-SP para cada modelo de veículo: um valor atingível pela maioria dos veículos daquele modelo, como o que deve ser exigido pelo cliente ou buscado pelo mecânico por ocasião da revisão do veículo; outro que identifica o início da desconformidade, o que justifica uma nova revisão; e um terceiro, intermediário, que possa indicar que a necessidade de manutenção não é imediata.

Este método também permite a geração de subsídios para a revisão dos valores de referênciaestabelecidos pelo CONAMA, aprimorando-os à medida que a sociedade adquire a conscientização e experiência necessárias ao seu comprometimento com as exigências ambientais. Também permite a identificação de modelos específicos que necessitem de limites diferentes em função das tecnologias que possuem, de forma a maximizar os benefícios do Programa, sem inviabilizar o seu atendimento pelos usuários.

\section{CHARACTERISTIC VALUES OF THE BRAZILIAN VEHICLE EMISSIONS REFERENCE PARAMETERS}

\section{Abstract}

This paper presents a statistical methodology for the identification of reference parameters effectively practiced of in inspection and maintenance programs- $/ / \mathrm{M}$, for verification of in-use vehicle emissions compliance withoriginal manufacturer specifications.

Three levels of emissionreference parameters were identified in theSão Paulo Cityl/M Program database for each vehicle model: a value attainable by most vehicles of that model which should be required by the client or sought by the repair shop for vehicle maintenance; anotheronethat identifies the beginning of non-conformity and thereforejustifies new service works; and a third that can indicate that service works are not required immediately.

This method also allows for the revision of the limits established by CONAMA and theirfine-tuningover timeas society gets the necessary experience and awareness to environmental demands.Also allows the identification of specific models that require different reference valuesdepending on the

\footnotetext{
${ }^{1}$ EnvironMentality - Consultor

${ }^{2}$ EnvironMentality - Consultor

${ }^{3} \mathrm{ADS}$ - Consultor

${ }^{4}$ CONTROLAR - Gerente de Engenharia do Produto

${ }^{5}$ Consultor
} 
technologies they have, in order to maximize the $\mathrm{I} / \mathrm{M}$ benefits, assuring the feasibility of vehicle maintenance and conformity.

\section{Introdução}

O Programa de Inspeção e Manutençãode Veículos da cidade de São Paulo (I/M-SP) produz anualmente mais de três milhões de dados de medições de emissões de veículos. Os resultados dessas inspeções compõem uma base de dados que cobre todas as marcas e modelos de veículos presentes na cidade, de forma que é possível extrair informações a respeito do comportamento típico de cada modelo de veículo em termos das suas características de emissão de poluentes.

Com base nos dados do Programa I/M-SP, opresente trabalho apresenta uma metodologia que possibilita obterdas estatísticas dos resultados da inspeção veicular ambiental valores característicos de emissão de cada modelo que indicamo estado de manutenção do veículo a fim de fornecer valores que permitam aos proprietários de veículosou mecânicosdeterminar a necessidade de efetuar a manutenção de seu veículo. Esses valores também podem ajudar asoficinas mecânicas obter a melhor regulagem possível para cada modelo de veículo e detectar a ocorrência de defeitos que porventura estejam afetando as emissões, bem como podem auxiliar os proprietários a verificar se os serviços de manutenção foram satisfatórios.As montadoraspodem utilizá-los paraverificar se algum dos modelos de sua produção apresenta comportamento anômalo em uso cotidiano, o que pode determinar o recolhimento do modelo para verificação e correção de defeitos ou a emissão de orientações específicas à sua rede de concessionários e demais oficinas mecânicas, contribuindo para a melhoria dasrevisões periódicas.

\section{Metodologia}

O objetivo deste trabalho é o de apresentar um novo conceito de utilização dos resultados de emissão dos veículos inspecionados em programas de inspeção e manutenção,possibilitando um uso mais ampliado desses resultados e benefícios para a sociedade como mencionado anteriormente.Neste sentido, considera-se que cada montadora deveria ter acesso às curvas de distribuição percentílica completas dos resultados de inspeção para os modelos de sua produção, enquanto que os proprietários de veículos deveriam poder ter acesso a alguns desses parâmetros estatísticos,como os percentis de 50\%,75\% e 90\% dessas curvas (p50, p75 e p90)para os seus modelos deveículos, para seu ano de fabricação e combustível.

Visando definir os valores característicos de emissão dos veículos em uso normal, as estatísticas foram elaboradas apenas sobre os valores de emissão provenientes de veículos aprovados no Programa I/M-SP, descartando-se com isso aqueles que apresentam defeitos graves de manutenção, que poderiam contaminar as estatísticas com valores que não são próprios do modelo em si. Dentre estes modelos, foram selecionados apenas aqueles com mais de 100 veículos aprovados nas inspeções de 2011 para garantia de ajuste e representatividade da distribuição percentílica daquele modelo. 
A definição dos valores característicos é baseada na distribuição percentílica das concentrações medidas dos poluentes considerados nos gases de escape - CO e HC para os veículos com motor do ciclo Otto (automóveis e motociclos) e opacidade da fumaça para os do ciclo Diesel - medidos conforme as metodologias oficiais, para cada modelo considerado. A figura 2.1 apresenta dois exemplos típicos dessas distribuições, neste caso para o monóxido de carbono.

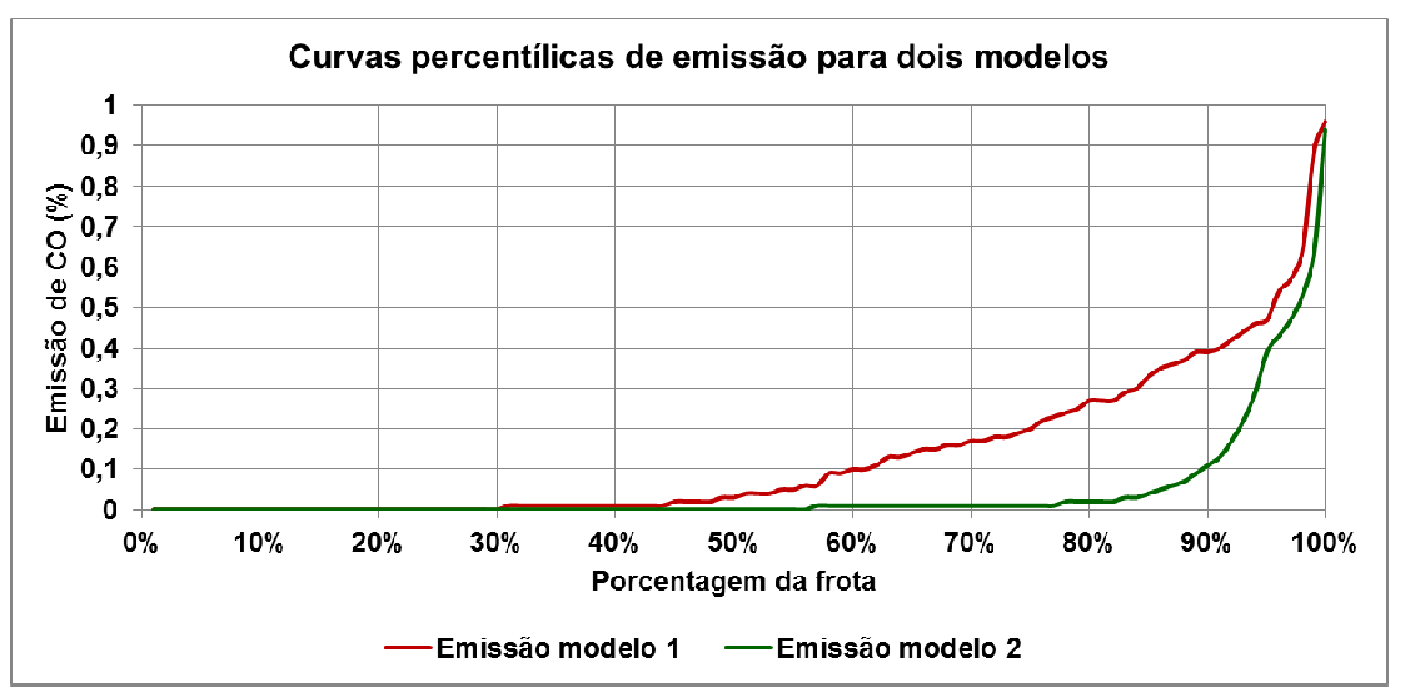

Figura 2.1: exemplo de curvas percentílicas da emissão de monóxido de carbono para dois modelos distintosde automóveis

Nestas curvas é possível verificar que, para o modelo1, cerca de $30 \%$ da frota possui emissão próxima de zero, $15 \%$ (entre os percentis de $30 \%$ e $45 \%$ ) possuem emissão ligeiramente acima de zero (na verdade, $0,01 \%$ de CO) e, a partir do percentil de $45 \%$, as emissões começam a subir gradativamente até o percentil de $95 \%$. Neste ponto da curva,o valor da emissão é de $0,47 \%$ de CO, o que significa que $95 \%$ da frotaaprovadadesse modelo possui emissão inferior a este valor. A partir do percentil de $95 \%$ as emissões elevam-se muito rapidamente, de forma que podese dizer que os cinco por cento da frota que possuem emissão acima de $0,47 \%$ de $\mathrm{CO}$ apresentam algum problema que influi na sua emissão, posto que este não é o comportamento característico do modelo considerado, afinal, 95\% emitem menos que esse valor.

Já para o modelo 2, a curva é bastante distinta em termos de valores, mas também existe uma certa porcentagem de veículos com emissão mínima, que neste caso atinge $56 \%$ da frota, um porcentual de veículos que possuem emissão muito baixa (faixa de p57\% a p82\%), uma parcela cujas emissões são crescentes, até $90 \%$ e 10\% de veículos cuja emissão cresce rapidamente, caracterizando possíveis problemas que poderiam ser melhorados por meio de uma revisão.

Esta análise, no entanto, poderia levar ao entendimento de que o modelo 1 fosse "melhor", em termos de emissão, do que o modelo 2, posto que para o modelo 1 apenas 5\% apresentam "problemas evitáveis"; entretanto, observa-se que ao longo de toda a curva os valores observados para o modelo 2 são inferiores, lembrando, ainda, que para esta análise foram considerados apenas os veículos que 
foram aprovados na inspeção, ou seja, veículos cujas emissões não ultrapassaram o limite de referência estabelecido pelo CONAMA, que neste caso era de $1 \%$ de CO.

Assim, define-se como tese deste trabalho a ideia de se estudar cada modelo em separado, não os comparando entre si, e partindo do princípio de que os exemplares estudados são veículos que não apresentam problemas sérios a ponto de infringirem a legislação vigente. O enfoque é, por outro lado, o de estabelecer "comportamentos normais", onde o veículo se apresentade acordo com seus pares em estado de manutenção normal e "comportamentos limítrofes", onde o veículo, embora ainda não venha a ser reprovado na inspeção, pois atende ao limite,já apresenta indícios de defeitos ou falhas de manutenção que o destacam em relação ao comportamento normalobservado na maioria da frota desse modelo.

A figura a seguir, baseada nos mesmos modelos da figura 2.1, mostra como será feita esta classificação.

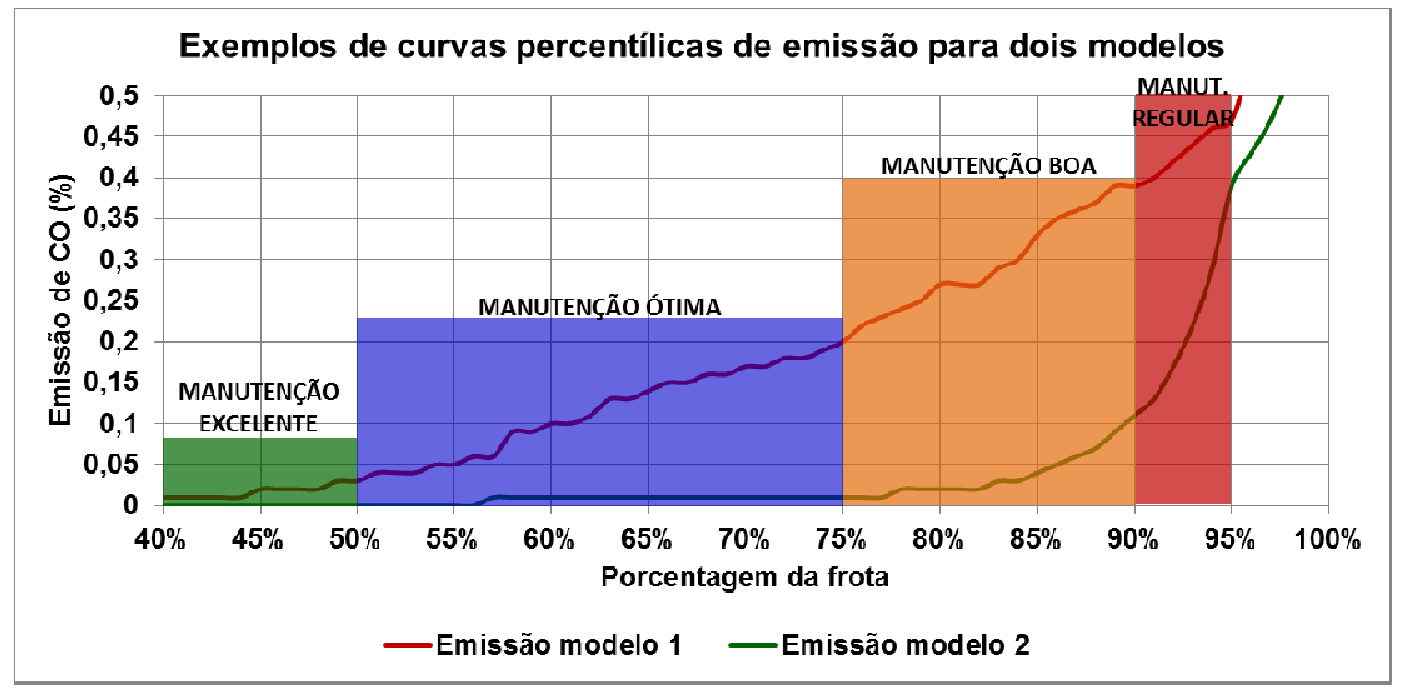

Figura 2.2: classificação do estado de manutenção conforme a distribuição percentílica - exemplo de monóxido de carbono

Com base neste tipo de gráfico, definem-se as classes de valores característicos como:

- excelentes - valores situados abaixo da mediana, representada pelo percentil de $50 \%$, ou p50, inclusive;

- ótimos - valores situados acima do p50 e abaixo do p75, inclusive;

- bons - valores situados acima do p75 e abaixo do p90, inclusive;

- regulares - valores situados acima do p90e abaixo do p95, inclusive.

É importante ressaltar que tais faixas referem-se exclusivamente ao estado de manutenção do veículo. Assim, o fato de um veículo do modelo 1 ser classificado como ótimo com uma emissão de $0,15 \%$ de $\mathrm{CO}$, enquanto que um veículo do modelo 2 com a mesma emissão é classificado como regular, não constitui incoerência do método, pois na verdade, o veículo do modelo 1 está sendo 
classificado como tendo nível de manutenção ótimo, o que significa que seu proprietário está mantendo seu veículo em ótimas condições dentro das possibilidades que este modelo oferece, enquanto que o veículo do modelo $2 \mathrm{com}$ emissão de $0,15 \%$ não está sendo tão bem mantido, pois o modelo oferece a possibilidade de emitir dez vezes menos, com a manutenção adequada.Em termos tecnológicos, as percentílicas são distintas para as diferentes fases do PROCONVE, daí a importância de separação por ano-fabricação e modelo.

Assim, no exemplo dado, os valores característicos destes dois modelos seriam os mostrados na tabela 2.1 .

Tabela 2.1: Valores característicos para o exemplo dado

\begin{tabular}{l|c|c|c|c|}
\hline \multirow{2}{*}{ Classificação } & \multicolumn{2}{|c|}{ Modelo 1 } & \multicolumn{2}{c|}{ Modelo 2 } \\
\cline { 2 - 5 } & V.C. inf & V.C. sup. & V.C. inf & V.C. sup. \\
\hline Excelente & 0,00 & 0,03 & 0,00 & 0,00 \\
\hline Ótimo & 0,03 & 0,20 & 0,00 & 0,01 \\
\hline Bom & 0,20 & 0,39 & 0,01 & 0,11 \\
\hline Regular & 0,39 & 0,47 & 0,11 & 0,39 \\
\hline $\begin{array}{l}\text { V.C. inf } \\
\text { V.C. sup. }\end{array}$ \\
\multicolumn{3}{|c|}{ Valor característico inferior da classe } \\
\hline
\end{tabular}

Em relação às classes de valores de referência, temos que:

- do ponto de vista do proprietário deve-se considerar que os veículos classificados como "manutenção regular" são aqueles para os quais é recomendado que se faça uma manutenção o quanto antes, pois encontramse em uma situação muito próxima de apresentar emissão em excessoe a grande maioria desse modelo ( $90 \%$ ou mais) emite quantidades inferiores; os classificados como "manutenção boa" são veículos que ainda não necessitam manutenção, mas é preciso atenção para que não venham a entrar na classe de "manutenção regular"; os de "manutenção ótima" e excelente são veículos que não apresentam problemas, estão de acordo com o projetado para 0 modelo e ano-fabricação;

- do ponto de vista de um mecânico, a faixa de valores classificada como de "manutenção excelente" é a que deve ser buscadapreferencialmentepara qualquer veículo que esteja em processo de manutenção embora os valores das faixas ótima e boa sejam igualmente aceitáveis. Entretanto, se estiver na faixa regular, deve-se buscar reduzir os valores de emissão para as faixas inferiores, pois $75 \%$ da frota desse modelo emite menos que o valor medido;

- do ponto de vista do fabricante, modelos que apresentemde forma frequente e consistentevalores de referência visivelmente superioresà faixa de bom estado de manutenção ou que resultem emcurvas percentílicas com formato anômalo ou que tenham comportamento muito diverso de outros veículos de mesmo padrão tecnológico, devem ser analisados em detalhe em busca das causas dasalterações observadas e de soluções para evita-las.Pode-se ainda 
validar o que foi projetado para o veículo em termos de fator de deterioração das emissões do modelo com o passar dos anos de uso.

Assim, as informações mais importantes a serem fornecidas para proprietários de veículos e oficinas mecânicas são os valores característicos de cada modelo. Já para os fabricantes, é interessante que estes tenham acesso ás curvas percentílicas completas, para que possam analisar o comportamento estatístico de cada um dos seus modelos, como mostra a figura 2.3.

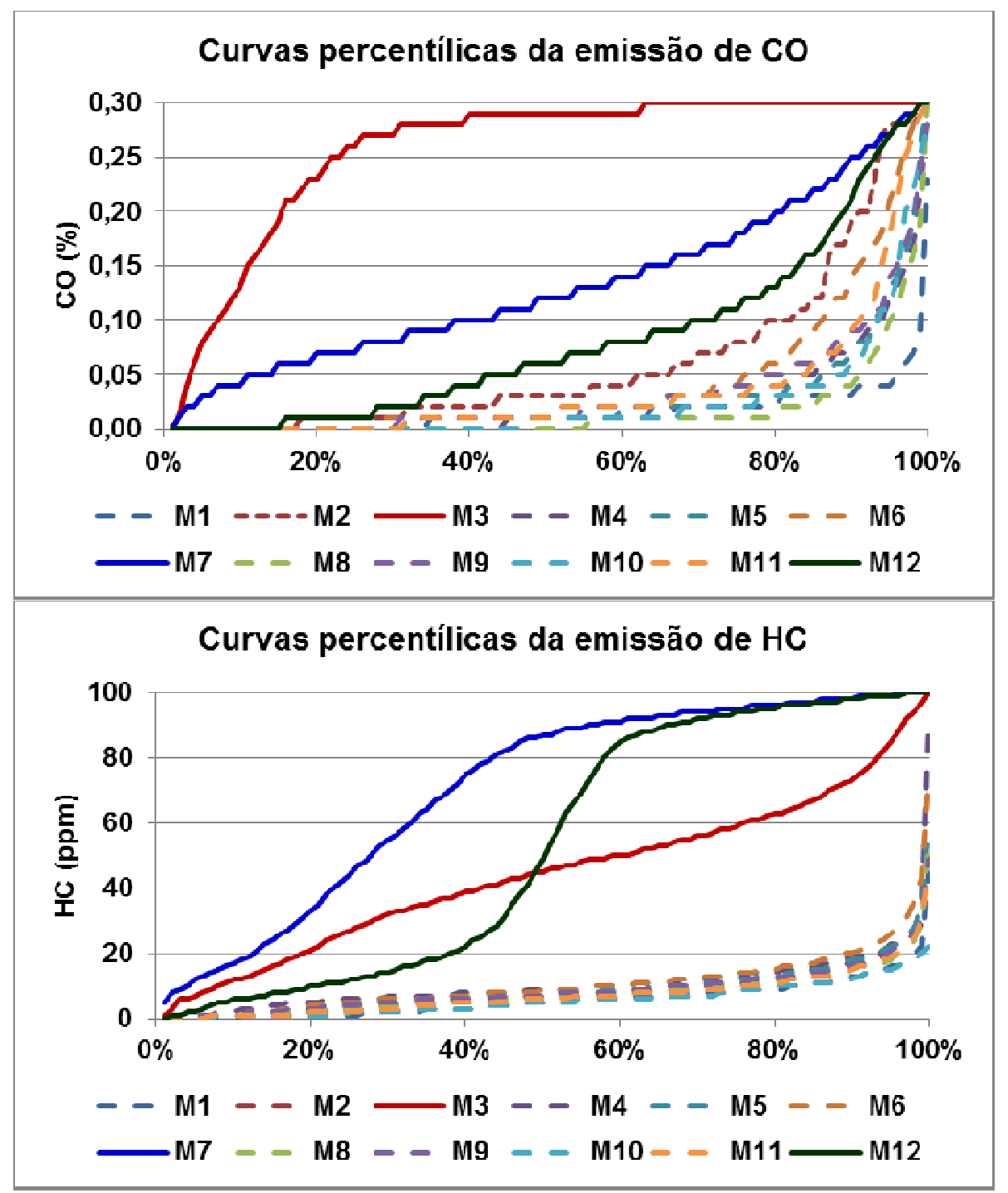

Figura 2.3: exemplo de emissão de diferentes modelos

Dentre os doze modelos selecionados na figura 2.3, observa-se que há três os modelos M3, M7 e M12 - que apresentam curvas percentílicas visivelmente distintas das curvas dos demais. Em relação às emissões de $\mathrm{CO}$, o modelo $\mathrm{M} 3$ apresenta uma curva invertida, com a curvatura para baixo, indicando que é possível atender ao limite, mas deixa dúvida se esta "conformidade" não estaria sendo obtida 
através de uma alteração das característicasnormais do veículo, que após a inspeção teria que ser desfeita por produzir efeitos indesejáveis, como piorar a dirigibilidade, aumentar consumo etc.É possível que este modelo possua algum tipo de defeito de fabricação ou mesmo de manutenção crônico. No primeiro exemplo, se for constatado pelo fabricante um defeito de fabricação ou de durabilidade de componentes que afetam o funcionamento do motor ou dos equipamentos de controle de emissões, o fabricante teria que determinar o recolhimento do veículo para reparação, sob pena de afetar a própria imagem da empresa. Caso o problema seja de manutenção, o fabricante deveria distribuir comunicados aos seus concessionários determinando como deve ser feita a manutenção especificamente para corrigir o problema detectado.

Fatos semelhantes são verificados para o modeloM7 em relação ao $\mathrm{HC}$. Embora ambos os modelos (M3 e M7) possuam alterações significativas de suas emissões tanto de $\mathrm{CO}$ como de $\mathrm{HC}$, os problemas aparentemente são distintos, pois as curvas percentílicas mostram padrões nitidamente diferentes.

No modelo M12 observam-se claramente dois comportamentos distintos na curva de HC. Comportamentos dessa natureza podem ser interpretados como decorrentes de alguma mudança tecnológica na produção do veículo que tenha modificado suas emissões ou seus fatores de deterioração. Neste caso, utilizado apenas a título de exemplo, trata-se de um modelo fabricado por vários anos,com mesma identificação no DENATRAN,porém atravessando mais de uma fase do PROCONVE, o que pode ter reduzido suas emissões de $\mathrm{HC}$ de um patamar em torno de 90 ppm para outro, ao redor de $10 \mathrm{ppm}$.

\section{Resultados}

Este trabalho foi feito com base em cerca de 1500 modelos de veículos do ciclo Otto, incluindo automóveis e utilitários, que abrangem todas as fases do PROCONVE, bem como modelos anteriores a esta legislação. Assim sendo, o banco de dados permite, além da avaliação de cada modelo em separado, como discutido no capítulo de metodologia, uma análise comparativa ampla, mostrando as diferenciações entre níveis tecnológicos e internas a cada nível.

Deve-se ter em mente que este trabalho não é feito com base em características derivadas de imposições legais, como fases do PROCONVE, que podem ser respeitadas com maior ou menor folga para cada modelo, ou mesmo antecipadas à legislação, como ocorreu com alguns modelos dotados de injeção eletrônica antecipando a fase L3. A base para este estudo são os valores medidosde emissão em marcha lenta ecujos padrões individuais são, muitas vezes, mais baixos do que o exigidonas fases do PROCONVE a que estão submetidos.

O exemplo a seguir (figura 3.1) mostra os valores característicos de todos os 524 veículos que atendem ao limite de $0,3 \%$ de $\mathrm{CO}$ (parte da fase L4 e a L5).Os modelos foram classificados pelo percentil p95 de $\mathrm{CO}$ e subdivididos em quatro gruposao longo da faixa de ocorrência desse parâmetro. Observa-se que o grupo G1, com 301 modelos, também apresenta baixa emissão de HC, exceto por dois modelos. O grupo G2 (79 modelos), ainda limitado ao nível de CO costumeiramente 
recomendado pelos fabricantes, de $0,1 \%$ de $\mathrm{CO}$, também apresenta emissão de $\mathrm{HC}$ em níveis mais baixos, exceto por cinco modelos. A partir do grupo G3 (79 modelos) e, especialmente o G4 (65 modelos), as dispersões aumentam e os comportamentos estatísticos se aproximam dos parâmetros de referência do CONAMA, tanto para $\mathrm{CO}$ quanto para $\mathrm{HC}$.

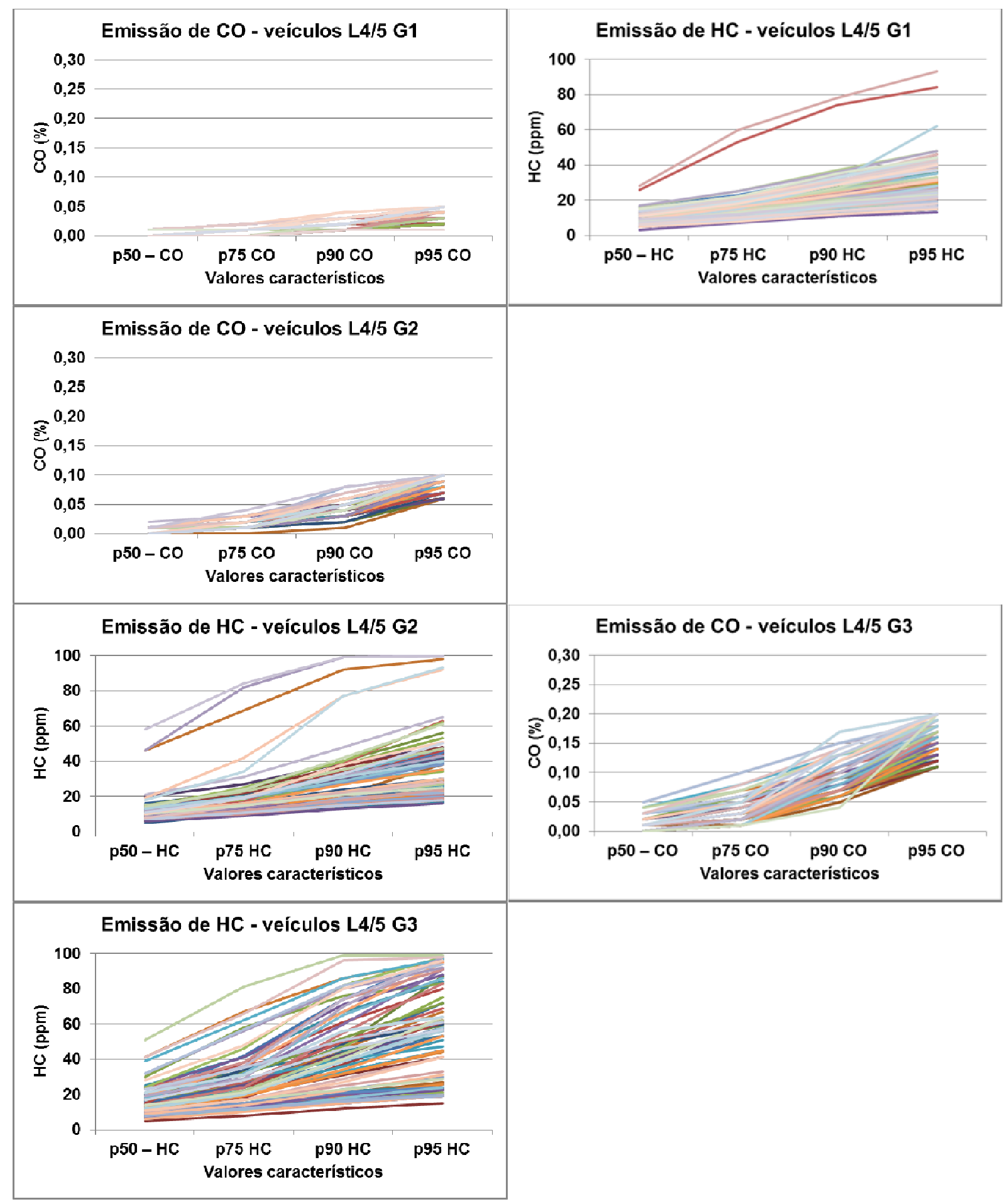




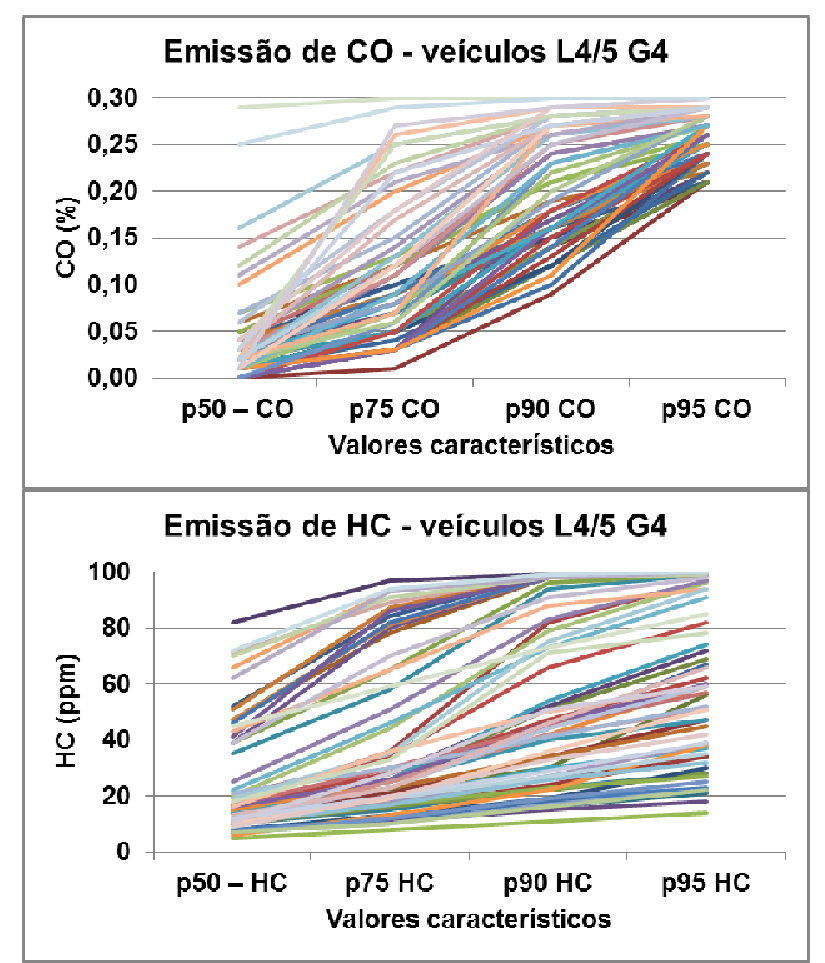

Figura 3.1 - Valores característicos de $\mathrm{CO}$ e $\mathrm{HCde}$ modelosque atendem aos limites de $0,3 \%$ de $\mathrm{COe} 100 \mathrm{ppm}$ de $\mathrm{HC}$, subdivididos em grupos em função do percentil p95 da emissão de CO

É importante salientar que as curvas superiores apresentam curvatura invertida (concavidade para baixo), indicando a possibilidade de dificuldades para a conformidade com os parâmetros de referência fixados pelo CONAMA, uma vez que a mediana ( $\mathrm{p} 50)$ também é alta.

Por esta abordagem pode-se verificar claramente que existem 380 modelos (301 do grupo G1 e 79 do grupo G2) que atendem aos valores de referência das fases 4 e $5(0,3 \%$ de $\mathrm{CO}$ e 100 ppm de $\mathrm{HC}$ ) com valores de emissão de $\mathrm{CO}$ muito abaixo do permitido, atingindo não mais do que $0,05 \%$ de $\mathrm{CO}$ no grupo G1 e $0,1 \%$ de $\mathrm{CO}$ no grupo G2. No caso do HC, à exceção de dois modelos do grupo G1 e 5 modelos do grupo G2, vale a mesma conclusão. Para esses dois grupos, fica evidente a oportunidade de revisar os valores de referência para aumentar a efetividade do Programa I/M sem prejuízo da viabilidade de atendimento pelos usuários.

Esta seria uma estratégia para revisar valores de referência com base nos valores característicos do conjunto de modelos de um mesmo nível tecnológico; outra estratégia seria a de adotar, da mesma forma como é feito para as emissões de fumaça dos veículos Diesel ou do ruído dos veículos Otto, valores de referência específicos para cada modelo em particular, desde que limitados ao valor máximo determinado para a fase do PROCONVE à qual pertence. 
Enquanto isso não é feito, uma campanha que vise a divulgação dos valores característicos pode trazer benefício semelhante, a partir da aceitação voluntária da população.

\section{Conclusões:}

A publicação de valores característicos da maioria dos modelos de veículos em circulação permite obter benefícios ambientais importantes, uma vez que produzem orientações fundamentais para otimizar o processo de manutenção visando os menores níveis de emissão possíveis para cada modelo. Além disso, geram subsídios para as montadoras reavaliarem o comportamento dos modelos sob sua responsabilidade em uso real baseado em estatísticas altamente consistentes.

Esta classificação orientará a tomada decisão de levar ou não o veículo para manutenção ou, estando em manutenção,verificar se o veículo possui emissões compatíveis com o que se espera deste modelo ou que seja definida uma ação de manutençãoadicional para reduzir suas emissões a um patamar inferior, de acordo com seus valores característicos, independente de qual seja ovalor de referência do I/M para aquela fase do PROCONVE, o qual é fixado para reprovar apenas os casos com defeitos mais graves, referentes a veículos que não apresentam condições para circulação.

Como resultado paralelo, a avaliação conjunta dos valores de referência de todos os modelos de uma determinada fase do PROCONVE permite a definição de grupos internos às fases para os quais podem ser definidos valores de referência mais condizentes com as necessidades ambientais e que sejam perfeitamente passíveis de serem atendidos. 